\title{
Characteristics of TIBr single crystals grown using the vertical Bridgman-Stockbarger method for semiconductor-based radiation detector applications
}

\author{
Dong Jin Kim ${ }^{1}$, JoOn-Ho OH ${ }^{1}$, Han Soo Kim ${ }^{1}$, Young Soo Kim ${ }^{1}$, MAnheE JeOng ${ }^{1}$, \\ Chang Goo KAng ${ }^{1}$, Woo Jin Jo ${ }^{1}$, HyojeOng Choi ${ }^{2}$, Jong Guk Kim ${ }^{1}$, Seung Hee LeE ${ }^{1}$, \\ JANG HO HA ${ }^{1, *}$
}

\footnotetext{
${ }^{1}$ Korea Atomic Energy Research Institute, 29 Geumgu-gil, Jeong-eup, Republic of Korea 580-185

${ }^{2}$ WCU Department of Energy Science, Sungkyunkwan University, Suwon, Republic of Korea, 440-746
}

\begin{abstract}
TlBr single crystals grown using the vertical Bridgman-Stockbarger method were characterized for semiconductor based radiation detector applications. It has been shown that the vertical Bridgman-Stockbarger method is effective to grow highquality single crystalline ingots of TlBr. The TlBr single crystalline sample, which was located $6 \mathrm{~cm}$ from the tip of the ingot, exhibited lower impurity concentration, higher crystalline quality, high enough bandgap $(>2.7 \mathrm{eV})$, and higher resistivity $\left(2.5 \times 10^{11} \Omega \cdot \mathrm{cm}\right)$ which enables using the fabricated samples from the middle part of the $\mathrm{TlBr}$ ingot for fabricating high performance semiconductor radiation detectors.
\end{abstract}

Keywords: semiconductor single crystal; TlBr; radiation detection; crystalline quality; impurity; resistivity; optical bandgap

(c) Wroclaw University of Technology.

\section{Introduction}

Semiconductor radiation detectors are of considerable technological importance due to their increasing use in medical imaging, and industrial and transportation security applications $[1,2]$. To fabricate such a high-quality semiconductor-based radiation detector, high-quality and high-purity semiconductor single crystals are needed. In addition, materials consisting of high atomic number elements are needed due to the higher probability of an incoming radiation beam interaction with valence electrons [3]. In this regard, a $\mathrm{TlBr}$ (atomic numbers: Tl-81, Br-35) compound semiconductor with a bandgap of $\sim 2.7 \mathrm{eV}$ is considered as one of the promising candidates for various applications, and was intensively researched by various research groups [4-6]. Among the various bulk crystal growth methods from the melt, zonemelting [7] and Bridgman-Stockbarger [4, 6, 8]

*E-mail: jha@kaeri.re.kr methods were used. In particular, the vertical Bridgman-Stockbarger method was shown to be superior due to the fact that it is the only method by which the large diameter $\mathrm{Tl}$ halide ingots can be produced [9].

Thus, in this paper, we report the fabrication and characterization of $\mathrm{TlBr}$ single crystals grown using the vertical Bridgman-Stockbarger method for use in radiation detector applications. In particular, the diced and polished samples were selected from the top, middle, and end parts of the ingot, and these samples were compared in terms of the structural, chemical, electrical, and optical properties.

\section{Experimental}

\subsection{Sample preparation}

$\mathrm{TlBr}$ raw powders of $99.998 \%$ purity were purchased from Alfa Aesar Co. and were used as-received. These $\mathrm{TlBr}$ powders were loaded into a precleaned quartz ampoule with a 1-inch 
diameter and vacuum sealed. This quartz ampoule was loaded into a homemade vertical BridgmanStockbarger furnace. This Bridgman-Stockbarger furnace consisted of a top zone, a sandwiched growth zone, and a bottom zone. After complete melting of the materials, the heater unit moved upward at a speed of $1 \mathrm{~mm} / \mathrm{h}$, and the temperature gradient at the growth zone was about $10{ }^{\circ} \mathrm{C} / \mathrm{cm}$. After the growth zone completely scanned the entire part of the ampoule, the grown ingot was then cooled down to room temperature for a cooling period of 10 hours, finally resulting in a 1inch single crystalline $\mathrm{TlBr}$ ingot with a length of about $95 \mathrm{~mm}$, as shown in Fig. 1a. This $\mathrm{TlBr}$ ingot was diced with a diamond wire saw to make the samples, as shown in Fig. $1 b$, and these samples were then mechanically polished with 800-, 2400-, 4000-grit SiC papers followed by chemical polishing/etching with an etching solution of mixed $\mathrm{HBr}$ and methanol (1:5). The samples, which were located about $3 \mathrm{~cm}, 6 \mathrm{~cm}$, $8 \mathrm{~cm}$ from the tip of the ingot, are herein referred to as the 'top', 'middle', and 'bottom' samples, respectively.

\section{Characterizations}

An impurity analysis was performed using an ICP-MS (inductively coupled plasma mass spectrometry) system (Nexion 300X, Perkin Elmer). Microstructural perfection of the samples was investigated using an XRD (X-ray diffraction) system (D/Max 4200, Rigaku) within the 2 theta range of 20 to 65 degrees. Optical transmittance results were obtained using a UV-Vis spectrophotometer (Cary 300, Agilent Technologies, Co.) equipped with an integrating sphere within the wavelength range of $200 \mathrm{~nm}$ to $800 \mathrm{~nm}$. The current-voltage (I-V) characteristics of the samples were measured with a parameter analyzer (4200SCS, Keithley). Before measuring the I-V data, $300 \AA$ thick gold-contacts were evaporated on both sides of the samples.

\section{Results}

Fig. 2 and Table 1 show the ICP-MS results for the raw material used, and the top, middle,
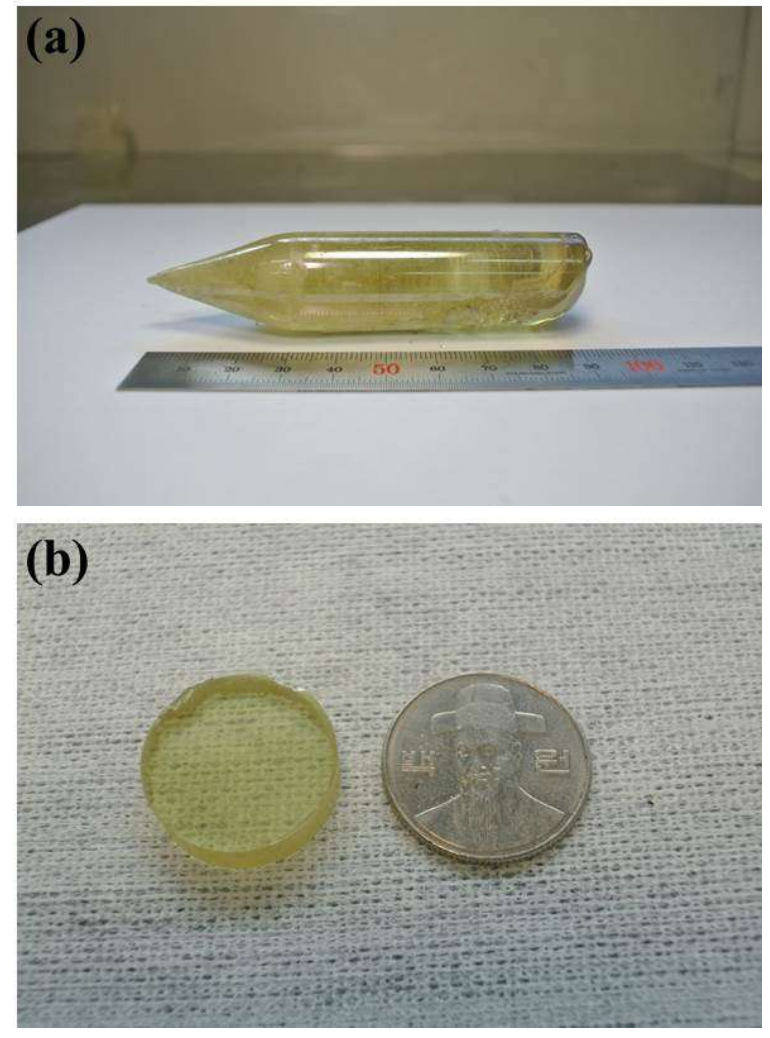

Fig. 1. (a) Single crystalline 1-inch $\mathrm{TlBr}$ ingot used in the study, (b) a diced and polished TIBr sample.

and bottom samples of $\mathrm{TlBr}$. It should be noted that the concentrations of the major impurity elements observed in the raw material were significantly reduced for the top and middle samples investigated in this study. More notably, some of the elements were not detected in the top, middle and bottom samples, possibly due to the detection limit (resolution) of the equipment used. In addition, the impurity $(\mathrm{Mg}, \mathrm{Fe}, \mathrm{Al})$ concentrations were higher in the bottom sample compared to those of the top and middle samples. For example, the concentrations of $\mathrm{Al}$ were $0.12 \mathrm{ppm}, 0.09 \mathrm{ppm}$, and $0.47 \mathrm{ppm}$ for the top, middle, and bottom samples, respectively. Similar purification effects using the BridgmanStockbarger technique were also reported by Dos Santos et al. [10].

Fig. 3 shows the XRD measurement results for the top, middle, and bottom samples investigated in this study. It should be noted that the middle sample (Fig. 3b) exhibits only a (llll 110$)$ peak 
Table 1. Summary of the major impurities observed in the raw material and in the three samples, which were measured using an ICP-MS.

\begin{tabular}{cccccccccc}
\hline Unit [ppm] & $\mathrm{Na}$ & $\mathrm{Ca}$ & $\mathrm{Mg}$ & $\mathrm{Fe}$ & $\mathrm{Al}$ & $\mathrm{Si}$ & $\mathrm{Ga}$ & $\mathrm{K}$ & $\mathrm{In}$ \\
\hline \hline Raw material & 2.00 & 5.00 & 0.20 & 3.00 & 0.50 & 2.00 & 0.20 & 0.10 & 0.10 \\
Top & 0.00 & 0.00 & 0.01 & 0.15 & 0.12 & 0.00 & 0.00 & 0.00 & 0.00 \\
Middle & 0.00 & 0.00 & 0.00 & 0.04 & 0.09 & 0.00 & 0.00 & 0.00 & 0.00 \\
Bottom & 0.00 & 0.00 & 0.04 & 0.22 & 0.47 & 0.00 & 0.00 & 0.00 & 0.00 \\
\hline
\end{tabular}

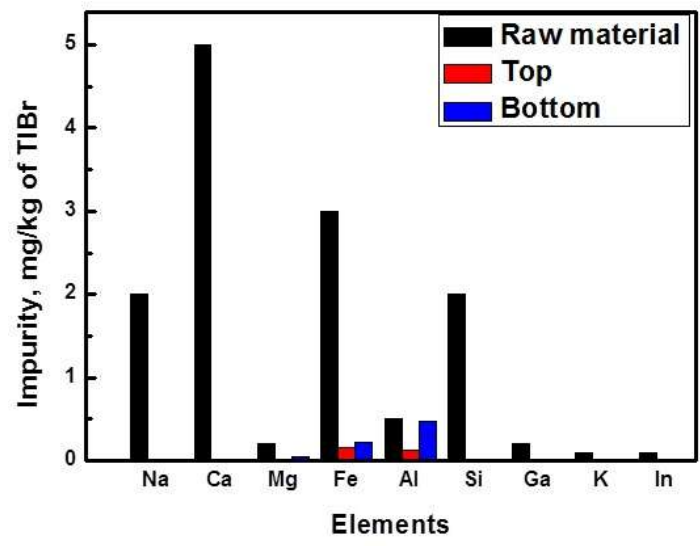

Fig. 2. ICP-MS results of the raw material, and top and bottom samples of $\mathrm{TlBr}$.

without any additional noticeable peaks within the measured range. However, the top sample additionally exhibits weak peaks corresponding to $\left(\begin{array}{lll}1 & 0 & 0\end{array}\right)$, (2 00 ), and (2 10 ) (Fig. 3a). More notably, the bottom sample shows a poorer crystalline quality compared to the other two samples (Fig. 3c).

Fig. 4a shows the transmittance curves for the three samples. It was shown that all the three samples exhibit similar absorption edges. From the transmittance data, optical bandgaps can be extracted. Park et al. explained [11] that the absorption coefficient $(\alpha)$ can be defined as:

$$
I=I_{0} \times \exp (-\alpha t)
$$

where $\mathrm{I}$ is intensity of the transmitted light, $\mathrm{I}_{0}$ - intensity of the incident light, $\mathrm{t}$ - thickness of a sample. In the direct transition semiconductor, $\alpha$ and optical energy bandgap $\left(E_{g}\right)$ can also be related by:

$$
\alpha=\left(h v-E_{g}\right)^{1 / 2}
$$

where $\mathrm{h}$ is Planck's constant, and hv is the photon energy of the incident light. Thus, after plotting $\alpha^{2}$ vs. hv curves, we can obtain optical bandgaps by extrapolating the linear part of the drawn curves to the $\alpha=0$ (x-axis), as can be seen in Fig. $4 \mathrm{~b}$. The optical bandgaps of $2.79 \mathrm{eV}$ (top), $2.76 \mathrm{eV}$ (middle), and $2.78 \mathrm{eV}$ (bottom) were obtained, which are all very similar to the reported value [4].

Fig. 5 shows I-V characteristics of the three samples with Au electrodes on both sides. All samples show very similar linear I-V characteristics, meaning that the $\mathrm{Au}$ electrode and $\mathrm{TlBr}$ formed ohmic contacts. It was calculated that the resistivities of the samples are $2.2 \times 10^{11} \Omega \cdot \mathrm{cm}$ (top), $2.5 \times 10^{11} \Omega \cdot \mathrm{cm}$ (middle), and $2.2 \times 10^{11} \Omega \cdot \mathrm{cm}$ (bottom), respectively.

\section{Discussion}

For any semiconductor material to be used in radiation detector applications that can be operable at room temperature, the material should have possibly low impurity concentration [12], the highest possible crystalline quality [13], a large bandgap, and a high resistivity [3] at the same time. In this regard, the data of Fig. 2 to Fig. 5, which are also summarized in Table 2, can be interpreted as follows. Defects including extrinsic point defects (impurities) and other crystalline defects can act as parasitic shunt paths for the charge carriers [14], leading to the higher leakage current and lower resistivities in the samples. Additionally, a higher amount of impurities in the bottom sample might be responsible for the deteriorated crystalline quality of the sample. In this connection, the middle sample can be judged to be more appropriate to make semiconductor radiation detectors due to the lower defect concentrations (Fig. 2), lower FWHM (full width at half-maximum) values with a higher 

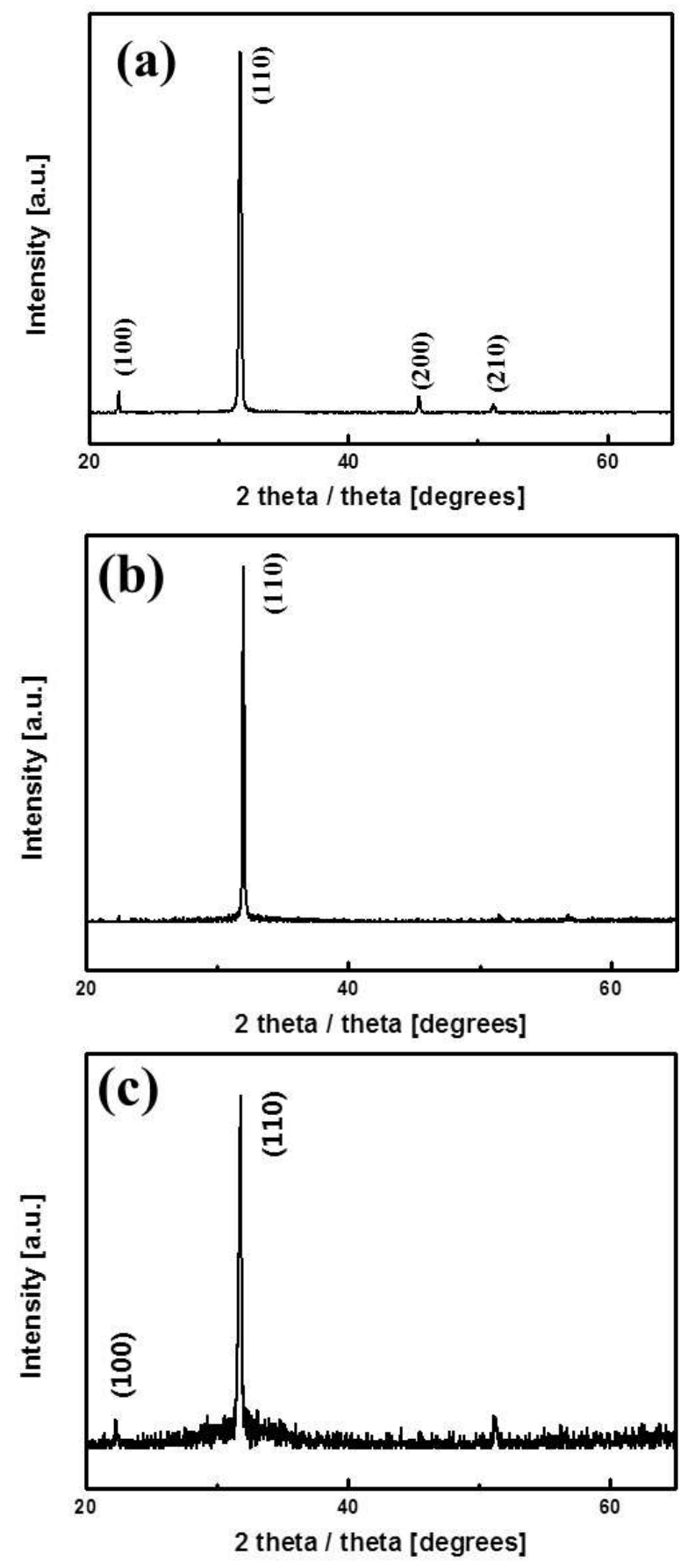

Fig. 3. XRD patterns for the top, middle, and bottom samples of $\mathrm{TlBr}$.

crystalline quality (Fig. 3), high enough bandgap (>2.7 eV, Fig. 4), and higher resistivity (Fig. 5). Thus, $\mathrm{TlBr}$ samples from the middle part of the ingot in this study can be regarded as one of the most promising materials for the next-generation radiation detectors.

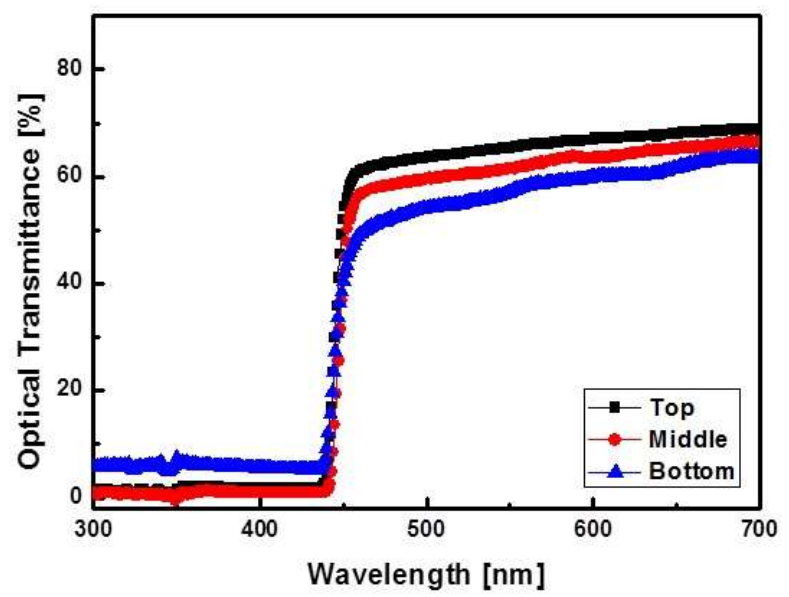

(a)

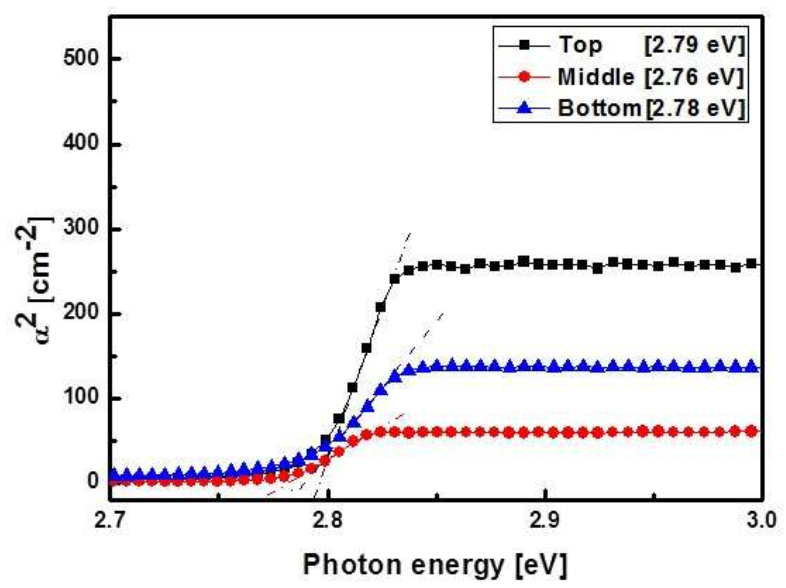

(b)

Fig. 4. (a) Transmittance and (b) $\alpha^{2}$ vs. hv curves for the top, middle, and bottom samples of TlBr.

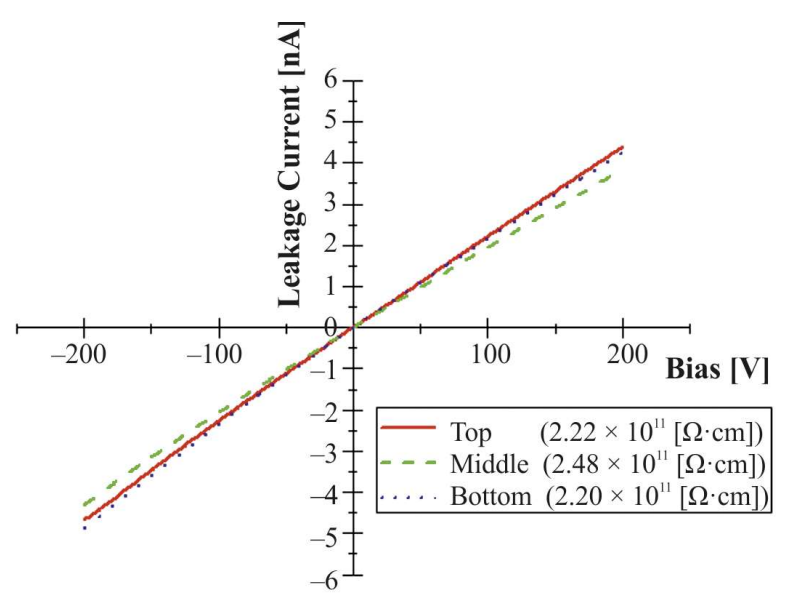

Fig. 5. I-V characteristics and resistivities of the top, middle, and bottom samples of $\mathrm{TlBr}$. 
Table 2. Summary of the Al impurity concentrations, FWHM (full width at half-maximum) values from the main peaks in the XRD graphs, optical bandgaps, and resistivities of the three samples used in this study.

\begin{tabular}{lccc}
\hline & Top & Middle & Bottom \\
\hline \hline Al impurity conc. [ppm] & 0.12 & 0.09 & 0.47 \\
FWHM [radian] & 0.215 & 0.156 & 0.223 \\
Optical bandgap [eV] & 2.79 & 2.76 & 2.78 \\
Resistivity $\left[\times 10^{11} \Omega \cdot \mathrm{cm}\right]$ & 2.2 & 2.5 & 2.2 \\
\hline
\end{tabular}

\section{Conclusions}

$\mathrm{TlBr}$ single crystalline samples for use in semiconductor radiation detection applications were fabricated using the vertical Bridgman-Stockbarger method. Based on the results of ICP-MS, XRD, optical bandgap, and I-V measurements, it can be seen that the middle sample has lower impurity concentrations, lower FWHM (full width at halfmaximum) values for a higher crystalline quality, high enough bandgap $(>2.7 \mathrm{eV})$, and higher resistivity $\left(2.5 \times 10^{11} \Omega \cdot \mathrm{cm}\right)$, which are all eligible properties for enhanced radiation detection properties. Therefore, $\mathrm{TlBr}$ single crystals can be regarded as one of the most promising materials for the next-generation semiconductor radiation detection device.

\section{Acknowledgements}

The authors would like to gratefully acknowledge the financial support by the Nuclear R\&D Program of the Ministry of Science, the ICT \& Future Planning (MSIP) of South Korea (NRF-2010-0026096, NRF-2013M2A2A4023359) and by a grant from the Korea Atomic Energy Research Institute (Grant No.: 523280-15).

\section{References}

[1] Szeles C., Phys. Status Solidi B, 241 (2004), 783.

[2] Nam H.G., Shin M.S., Cha K.H., Cho N.I., Yun E.J., J. Korean Phys. Soc., 48 (2006), 1514.

[3] Androulakis J., Peter S.C., Li H., Malliakas C.D., Peters J.A., LiU Z., Wessels B.W., Song J.-H., Jin H., Freeman A.J., Kanatzidis M.G., Adv. Mater., 23 (2011), 4163.

[4] Kouznetsova M.S., Lisitskya I.S., ZatoloKaB S.I., Gostilo V.V., Nucl. Instrum. Meth. A, 531 (2004), 174.

[5] Нitomi K., Shoji T., Ishiı K., J. Cryst. Growth, 379 (2013), 39.

[6] Shorohov M., Muktepavela F., Grigorjeva L., Maniks J., Millers D., Nucl. Instrum. Meth. A, 607 (2009), 120.

[7] Hitomi K., Kikuchi Y., Shoj T., IshiI K., IEEE T. Nucl. Sci., 56 (2009), 1859.

[8] Kostamo P., Shorohov M., Gostilo V., Sipila H., Kozlov V., Lisitsky I., Kuznetsov M., LANKINEN A., DANILEWSKY A.N., LiPSANEN H., LESKELA M., Nucl. Instrum. Meth. A, 607 (2009), 129.

[9] Kozlov V., Andersson H., Gostilo V., Kemell M., Kostamo P., Kouznetsov M.S., LESKELA M., LiPSANEN H., LisitSKY I.S., SHOROHOV M., SiPILA H., Nucl. Instrum. Meth. A, 607 (2009), 126.

[10] Santos Dos R.A., Silva DA J.B.R., GENNARI R.F., Martins J.F.T., Ferraz C.D.M., Hamada M.M., MESQUITA DE C.H., in Proceedings of the IEEE Nuclear Science Symposium and Medical Imaging Conference, Anaheim, 2012.

[11] PARK K.C., MA D.Y., Kim K.H., Thin Solid Films, 305 (1997), 201.

[12] Hitomi K., Onodera T., Shoji T., Nucl. Instrum. Meth. A, 579 (2007), 153.

[13] Hitomi K., Matsumoto M., Muroi O., Shoji T., Hiratate Y., J. Cryst. Growth, 225 (2001), 129.

[14] Dyk VAn E.E., Meyer E.L., Renew. Energ., 29 (2004), 333. 